\title{
Efficacy of Emergency Severity Index (ESI) in Early Identification of Patients with Sepsis and Septic Shock at Triage
}

\section{Emad Uddin Siddiqui}

Aga Khan University Hospital

\section{Noman Ali}

Aga Khan University Hospital

Mirza Noor Ali Baig ( $\nabla$ noor.baig@aku.edu )

Aga Khan University Hospital https://orcid.org/0000-0002-6575-7237

Ahmed Raheem

Aga Khan University Hospital https://orcid.org/0000-0003-4367-1042

Nazir Najeeb

Aga Khan University Hospital

\section{Feroza Perveen}

Aga Khan University Hospital

\section{Kazi Khalid}

Aga Khan University Hospital

\section{Research note}

Keywords: Emergency department, Triage, Sepsis, Septic Shock Emergency severity index

Posted Date: September 10th, 2020

DOl: https://doi.org/10.21203/rs.3.rs-67848/v1

License: @ (1) This work is licensed under a Creative Commons Attribution 4.0 International License. Read Full License 


\section{Abstract}

Objectives The objective of the study is to determine the efficacy of Emergency Severity Index (ESI) tool in early recognition of "sepsis" and "septic shock" at the triage of an emergency room at a tertiary care hospital in Pakistan.

Results A total of 240 patients were included in this study. The area under the curve (AUC) for the ESI score I for septic shock was 0.943 [0.921-0.964] with the optimal cutoff value of 2.0 with sensitivity of $88.5 \%$ and specificity of $100 \%$. Similarly, the sensitivity and specificity of ESI score II for the diagnosis of sepsis was found to be $100.00 \%$ (Cl $97.63-100.00 \%)$ and $66.28 \%$ (Cl $55.28 \%$ to $76.12 \%)$ with accuracy of $87.92 \%$ (83.11-91.76\%)

\section{Introduction}

Sepsis is life-threatening organ dysfunction due to a dysregulated host response to infection and an important health problem. ${ }^{1}$ Sepsis is a global health problem contributing to high mortality rates as highlighted by several studies. In 2017, the global estimates for sepsis incidence were around 49 million cases, with approximately $19.7 \%$ deaths, this increases to $55 \%$ of all in-hospital sepsis related deaths. ${ }^{2}$ Data from emergency rooms of USA account for approximately 500,000 ED visits annually with suspected sepsis. ${ }^{3}$ With each hour delay in effective antimicrobial administration, the mortality of sepsis is expected to increase by $7.6 \% .{ }^{4} \mathrm{~A}$ significant component of sepsis-related deaths is attributed to "septic shock" which is characterized by persistent hypotension requiring vasopressors and hyper-lactatemia. The mortality from septic shock ranges from $24-41 \%{ }^{5}$

Across the globe huge sepsis burden are from low and middle -income countries, and $90 \%$ deaths attributable from pneumonia, meningitis or other infections from developing countries and majority of deaths dueto sepsis occur in Asia and sub-Saharan Africa. ${ }^{6}$ In Pakistan, the annual incidence of sepsis was $1600-2500$ per 100,000 , with $30 \%$ mortality rate. ${ }^{7}$

Considering the burden and significant mortality rates associated with sepsis, these patients must be prioritized and managed immediately in order to prevent adverse outcomes including death. To minimize the door to treatment time is even more challenging in overcrowded Emergency Departments (EDs) especially in resource-limited settings like Pakistan. The ED overcrowding and choked throughput is directly related with inadequate management of septic patients like delay in administration of antibiotics. ${ }^{8} \mathrm{Hence}$ it is necessary to introduce a purposeful and effective triage tool for ED nurses and physicians to prioritize and manage the patients according to the severity of their illness. ${ }^{9}$

Emergency severity index (ESI), a widely used tool that was developed in order to assess illness severity, hence to identify the sick patients that need immediate attention at triage. The ESI algorithm is a reliable tool with 4 decision points which provide good assistance with regard to early and reliable identification of patient with sepsis. (Fig.S1) 
In ESI, patients are either assigned to one of the five triage categories, ESI level 1 being the most acute and life threatening, while ESI level 5 being the least acute triage category, however patients with ESI levels 1 and 2 are those critical patients who should not wait to be seen and treated. ${ }^{1}$ Patient in category 2 are the one who are clinically unstable (septic or not) and category 3 are one with abnormal vitals. ${ }^{1}$

This study is aimed to identify the efficacy of ESI tool in early recognition of patients with sepsis and septic shock presented at the triage of our emergency department to help minimize the delay in appropriate management of these patients.

\section{Methods}

This is a single center, cross-sectional study conducted at the Emergency Department of the Aga Khan University Hospital. The university hospital is tertiary care teaching center that provides emergency care to over 75,000 patients per year. Ethical approval from ethics review committee of the Aga Khan University was taken to conduct the study.

All adult patients ( $\geq 18 y r$ ) presented to the emergency department over a period of six months from December 2016 to May 2017 with emergency diagnosis of sepsis and septic shock were enrolled. We enrolled cases through non-probability consecutive sampling via calculating the prevalence (7\%) of sepsis in Pakistan. Calculating with 95\% confidence interval (CI), 3\% margin of error and 0.05 level of significance, with WHO sample size calculator the estimated sample size was 240 . Patients were followed by reviewing their electronic medical records through patient care information software where patient medical diagnosis and discharge diagnosis were present.

Exclusion criteria was pregnancy, poly-trauma, patient had major surgery in past 30 days, prior do-notresuscitate orders or known chronic deliberated patient like new onset cerebrovascular accident and seizure disorder. Data was collected on a predesigned data sheet. No study-related therapeutic or diagnostic interventions were carried out.

Data was analyzed on SPSS $v$ 20. Descriptive and Demographics results were presented in term of Mean \pm $\mathrm{SD}$, frequency and percentages. Utility of Emergency Severity Index (ESI) in Adults with Sepsis were the unit of analysis Proportions and percentage was calculated for gender, sepsis and septic shock. Analysis on ROC area under curve was calculated to see overall performance of ESI in diagnosing sepsis and septic shock with 95\% confidence interval $(\mathrm{Cl})$. The results have been analyzed using the R-programming software version (3.5.2). Sensitivity and specificity of ESI was also assessed.

\section{Results}

A total of 240 patients were included in this study, out of which $57.9 \%$ (139) were male. The mean age was $52.7 \pm 15.3$ years with more than $59.6 \%$ above 50 years of age. Emergency severity index criteria comprises of 24.2\% (58) ESI I, 64.2\% (154) ESI II and 11.6\% (28) ESI III patients. Mean length of hospital stay was 2.2 \pm 1.1 . Out of total 240 patients, 183 patients were diagnosed with sepsis and 57 with septic shock. 179 
(74.6\%) patients were recovered and discharged. Most of the discharged patients (87.1\%) were belong to sepsis category. The mortality was found to be $19.6 \%$ (47 patients) out of the total study sample of which $63.8 \%$ (30) patient were belong to septic shock category and it was statistically significant. Fourteen patients were left against medical advice. Patients belong to sepsis category were more elderly as compared to septic shock (P-value $<0.001^{*}$ ). $65 \%$ (156) of patients with the diagnosis of sepsis were recovered and discharged and that was statistically significant (P-value $<0.001 *$ ). Demographic and clinical characteristics of the enrolled patients stratified by Sepsis and Septic shock are presented in Table 1.

Table-1: Demographic and clinical characteristics stratified by Sepsis and Septic shock 


\begin{tabular}{|c|c|c|c|c|}
\hline & Sepsis & Septic Shock & Total & \multirow[t]{3}{*}{ P-value } \\
\hline & {$[\mathrm{n}=183]$} & {$[\mathrm{n}=57]$} & {$[\mathrm{n}=240]$} & \\
\hline & $(\%) \mathrm{f}$ & $(\%) \mathrm{f}$ & $(\%) \mathrm{f}$ & \\
\hline \multicolumn{5}{|l|}{ Age Groups } \\
\hline$<=50$ Years & $35 \%(84)$ & $5.4 \%(13)$ & $40.4 \%(97)$ & \multirow[t]{3}{*}{$0.002 *$} \\
\hline$>50$ Years & $41.3 \%(99)$ & $18.3 \%(44)$ & $59.6 \%(143)$ & \\
\hline Total & $76.3 \%(183)$ & $23.8 \%(57)$ & $100 \%(240)$ & \\
\hline \multicolumn{5}{|l|}{ Length Hospital } \\
\hline$<=2$ Days & $49.6 \%$ (119) & $13.8 \%(33)$ & $63.3 \%(152)$ & \multirow[t]{3}{*}{0.329} \\
\hline$>2$ Days & $26.7 \%(64)$ & $10 \%(24)$ & $36.7 \%(88)$ & \\
\hline Total & $76.3 \%(183)$ & $23.8 \%(57)$ & $100 \%(240)$ & \\
\hline \multicolumn{5}{|l|}{$\underline{\text { Gender }}$} \\
\hline Male & $45.8 \%(110)$ & $12.1 \%(29)$ & $57.9 \%(139)$ & \multirow[t]{3}{*}{0.218} \\
\hline Female & $30.4 \%(73)$ & $11.7 \%(28)$ & $42.1 \%(101)$ & \\
\hline Total & $76.3 \%(183)$ & $23.8 \%(57)$ & $100 \%(240)$ & \\
\hline \multicolumn{5}{|l|}{ ESI Criteria } \\
\hline ESI I & $0.4 \%(1)$ & $23.8 \%(57)$ & $24.2 \%(58)$ & \multirow[t]{4}{*}{$<0.001 *$} \\
\hline ESI II & $64.2 \%(154)$ & $0 \%(0)$ & $64.2 \%(154)$ & \\
\hline ESI III & $11.7 \%(28)$ & $0 \%(0)$ & $11.7 \%(28)$ & \\
\hline Total & $76.3 \%(183)$ & $23.8 \%(57)$ & $100 \%(240)$ & \\
\hline \multicolumn{5}{|l|}{ Disposition } \\
\hline Critical Area & $41.3 \%(99)$ & $11.7 \%(28)$ & $52.9 \%(127)$ & \multirow[t]{4}{*}{0.755} \\
\hline Resuscitation & $14.6 \%(35)$ & $4.6 \%(11)$ & $19.2 \%(46)$ & \\
\hline Step Down & $20.4 \%(49)$ & $7.5 \%(18)$ & $27.9 \%(67)$ & \\
\hline Total & $76.3 \%(183)$ & $23.8 \%(57)$ & $100 \%(240)$ & \\
\hline \multicolumn{5}{|l|}{ Fate } \\
\hline Discharged & 65\% (156) & $9.6 \%(23)$ & $74.6 \%(179)$ & \multirow[t]{4}{*}{$<0.001 *$} \\
\hline Expired & $7.1 \%(17)$ & $12.5 \%(30)$ & $19.6 \%(47)$ & \\
\hline Left against medical advice & $4.2 \%(10)$ & $1.7 \%(4)$ & $5.8 \%(14)$ & \\
\hline Total & $76.3 \%(183)$ & $23.8 \%(57)$ & $100 \%(240)$ & \\
\hline
\end{tabular}


Sepsis was taken as reference category. The area under the curve (AUC) for the ESI score I for septic shock was 0.943 [0.919-0.996] with the optimal cutoff value of 2.0 with sensitivity of $88.5 \%$ and specificity of $100 \%$ Table 2 \& Fig. 1. Similarly, the sensitivity and specificity of ESI score II for the diagnosis of sepsis was found to be $100.00 \%$ (Cl $97.63-100.00 \%$ ) and $66.28 \%$ (CI $55.28 \%$ to $76.12 \%$ ) with accuracy of $87.92 \%$ (83.11-91.76\%)

Table-2: AUC, Sensitivity and specificity of Validation of Emergency severity index (ESI score I) at triage with septic shock

\begin{tabular}{|c|c|}
\hline ESI Criteria & Statistics \\
\hline Cutoff & 1.5 \\
\hline AUC (95\% CI) & (95\% C.I: 0.919- 0.996) \\
\hline P-value & $<0.001^{*}$ \\
\hline TP & 162 \\
\hline FP & 0 \\
\hline TN & 57 \\
\hline FN & 21 \\
\hline $\begin{array}{c}\text { TP proportion } \\
\text { (Sensitivity) }\end{array}$ & 0.885 \\
\hline $\begin{array}{c}\text { TN proportion } \\
\text { (Specificity) }\end{array}$ & 1 \\
\hline
\end{tabular}

\section{Discussion}

Early recognition of patients with sepsis, defined as a life threatening organ dysfunction due to a dysregulated host response to infection, remains a major challenge. Our study showed a mortality of $19.6 \%$ in patients with sepsis which is in alignment with the global mortality of $19.7 \% .{ }^{2}$ In addition the mortality from septic shock in our study was $63.8 \%$ which is almost double the mortality rate reported in some other studies. ${ }^{5}$ The Surviving Sepsis Campaign 1-h bundle stressed once more on the importance of timesensitive measures. In this single centered prospective study, we statistically validated the relevance of ESI as a tool to identify and screen the patients with sepsis and septic shock. We found out that ESI category I was $88.5 \%$ sensitive and $100 \%$ specific in identifying septic shock at triage (Table-2). This was probably due to the hemodynamic instability of the patients with septic shock on presentation at triage that led to their categorization in ESI for immediate intervention and treated as a life-threatening condition. This could have helped in timely management of these patients in the initial 1st hour which is much crucial and critical period, and may have major detrimental effect on the clinical outcome. 
Our study also showed $100 \%$ sensitivity and $66.28 \%$ specificity of ESI score II in diagnosing patients with sepsis. A study done by R. Nieves Ortega et al showed that ESI score of $\leq$ III was $32.5 \%$ specific and $97.4 \%$ sensitive in identifying sepsis with high number of false positives.

Another study done by Geier F. et.al, and in their study ESI didn't show the high values of diagnostic accuracy in identifying patients with sepsis (AUC 0.609; 95\% Cl: 0.704-0.853). This can be due to variable presentation with regards to presenting complains and vital signs of the patients They gave an example of 73-year-old male who visit ED with fever of $39.2^{\circ} \mathrm{C}$, cough and yellow discoloration. This case had history of organ transplantation, but was not identified at triage assessment. As per the ESI decision rule, he was assigned ESI III, and was waited for minutes or hours before been seen by physician. Hence Geier F et. al. identified that ESI triage system has weakness with regards to early identification of patient with sepsis, however patient with septic shock are able to identified reliably as with our results ${ }^{12}$

Our study showed that $11.6 \%$ of the patients were categorized as ESI III, which is a high number considering the significant mortality associated with sepsis and further studies are required to assess the factors associated with the variation in categorization of these patients in ESI III and above.

We can recommend that ESI application at the triage is useful in the prioritization of patients with sepsis and septic shock. Further studies will be required to compare ESI with other scoring systems and triage tools in order to compare their efficacy.

\section{Conclusion}

Sensitive and simple decision tools are required to promptly identify patients at risk of sepsis, as waiting times and resource allocation rely mostly on clinical information alone. Sepsis and septic shock is one of the major concerns in the lower to middle income country and is one of the major diagnoses responsible for morbidity and mortality in patients presenting to emergency departments. Identifying such patients in whom the first hour is claimed to be a golden hour and is decisive of patient's outcome is important and life saving measure. Having a reliable triage tool is of paramount importance which enables emergency physicians not to miss out any patient with sepsis and septic shock

In our study, ESI proved to be a useful "triage-tool" with high sensitivity and specificity in the identification and prioritization of patients with sepsis and septic shock in a busy emergency department. We were able to identify all patients with septic shock as ESI category I and most of the patients with sepsis as ESI category II. This would help physicians in managing the sickest patient first in order to improve outcomes in terms of mortality.

\section{Limitations}

One of the major limitations of our study is that we did not compare ESI with other scores like APACHE or SOFA scores that may have provided us with robust inferences. In addition, the analysis may have been 
influenced by the revised definitions of sepsis and septic shock as we merged the patients previously diagnosed as severe sepsis with those diagnosed as sepsis as per the modified definitions.

\section{List Of Abbreviations}

ESI: Emergency Severity Index

\section{Declarations}

Ethics approval and consent to participate: Attached

Consent for publication: Not Applicable

Availability of data and material: The data is the property of The Aga Khan University and any decision for the acquisition of data will be made by the desired authorities (HIMS, the AKUH).

Competing interests: Not Applicable

Funding: Not Applicable

Author's contributions:

1-Emaduddin Siddiqui : Conception \& Design

2- Noman Ali: Design \& Analysis

3- Mirza Noor Baig: Interpretation of data, revision of the work, correspondence

4- Ahmed Rahim: Analysis \& Interpretation

5- Nazir Najeeb: Drafting, Acquisition of data

6- Kazi Khalid: Acquisition of data

7- Feroza Parveen: Acquisition of data.

Acknowledgements: Not Applicable

\section{References}

1. Singer M, Deutschman CS, Seymour CW, et al. The third international consensus definitions for sepsis and septic shock (Sepsis-3). JAMA 2016; 315: 801-10.

2. Fleischmann C, Scherag A, Adhikari NKJ, et al. Assessment of global incidence and mortality of hospital-treated sepsis: current estimates and limitations. Am J RespirCrit Care Med 2016; 193: 25372. 
3. Peltan ID, Bledsoe JR, Oniki TA, Sorensen J, Jephson AR, Allen TL, Samore MH, Hough CL, Brown SM. Emergency department crowding is associated with delayed antibiotics for sepsis. Annals of emergency medicine. 2019 Apr 1;73(4):345-55.

4. Wang HE, Shapiro NI, Angus DC, Yealy DM. National estimates of severe sepsis in United States emergency departments. 2007; 35(8):1928-1936.

5. Ortega R N RC, Bingisser R, Christian H. Nickel. Clinical scores and formal triage for screening of sepsis and adverse outcomes on arrival in an emergency department all-comer cohort. JEmergMed. 2019;0ct57(4):453-60.e2.

6. Cheng AC, West TE, Limmathurotsakul D, Peacock SJ. Strategies to reduce mortality from bacterial sepsis in adults in developing countries. PLoS Med 2008;5(8):e175

7. Rudd K E, Johnson S C, Agesa K M, Shackelford K A, Tsoi D, Kievlan D R,et.al. Global, regional, and national sepsis incidence and mortality, 1990-2017: analysis for the Global Burden of Disease Study. Lancet 2020; 395: 200-11

8. Kumar A, Roberts D, Wood KE, et al. Duration of hypotension before initiation of effective antimicrobial therapy isthe critical determinant of survival in human septic shock.Crit Care Med. 2006; 34:1589-96.

9. Gilboy N, Tanabe T, Travers D, Rosenau AM. Emergency Severity Index (ESI): A Triage Tool for Emergency Department Care, Version 4. Implementation Handbook 2012 Edition. AHRQ Publication No. 12-0014. Rockville, MD. Agency for Healthcare Research and Quality. November 2011. Download on July 15, 2020.

https://www.ahrq.gov/sites/default/files/wysiwyg/professionals/systems/hospital/esi/esihandbk.pdf

10. Khan NU, Razzak JA, Alam SM, Ahmad H. Emergency department deaths despite active management: experience from a tertiary care centre in a low-income country. Emerg Med Australas.2007;19(3):213-7.

11. Grossmann FF, Nickel CH, Christ M, Schneider K, SpirigR,Bingisser R. Transporting clinical tools to new settings: culturaladaptation and validation of the Emergency Severity Index inGerman. Ann Emerg Med 2011;57:257-64.

12. Geier F PS, Greve Y, Achterberg A, Glöckner E. et.al. Severity illness scoring systems for early identification and prediction of in-hospital mortality in patients with suspected sepsis presenting to the emergency department. Wien Klin Wochenschr. 2013, Sept;125(17-18):508-15:8.

\section{Figures}




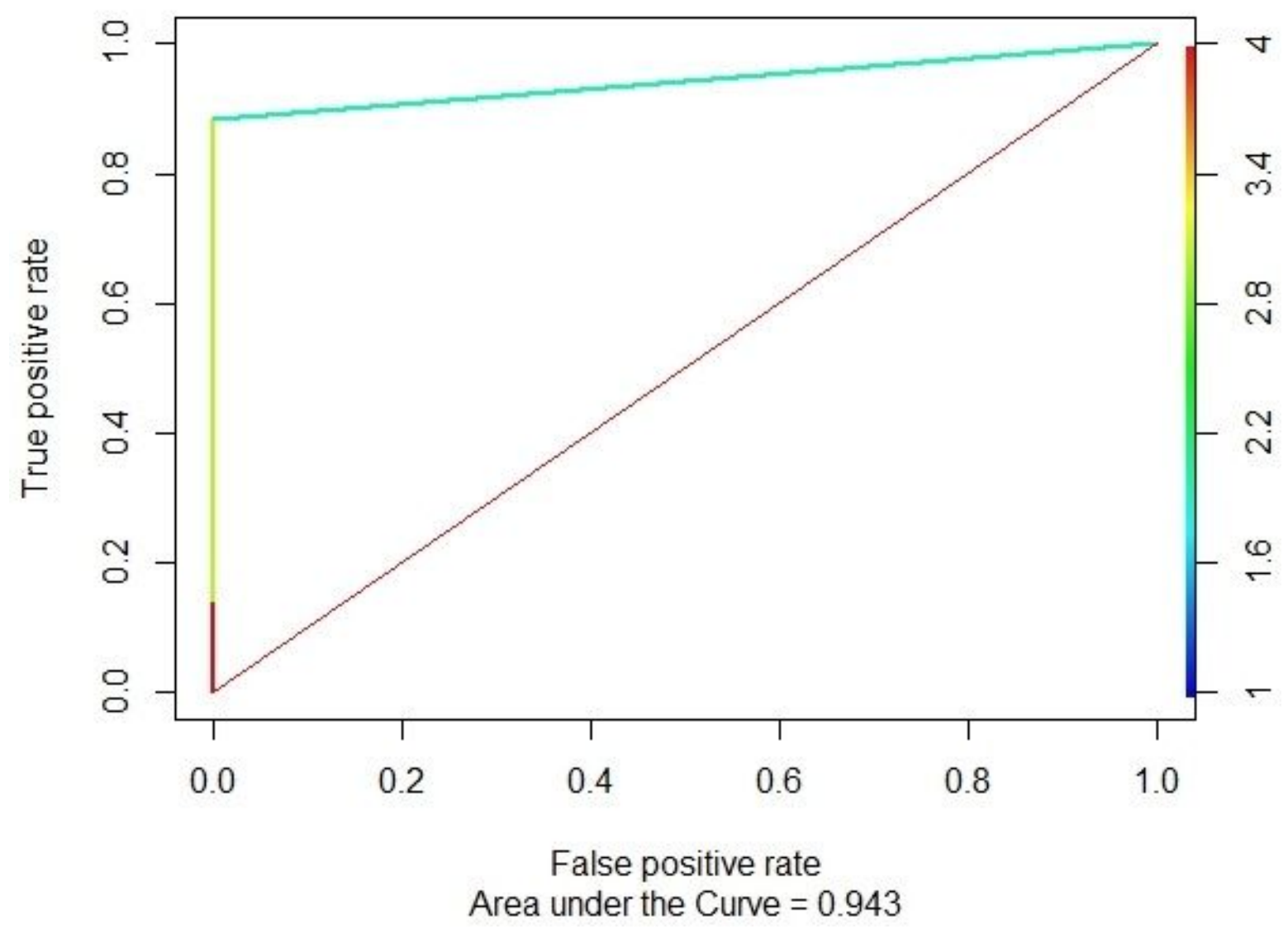

Figure 1

Validation of Emergency severity index (ESI) I with Septic Shock 